\title{
Mechanical Properties of Nanoporous Gold in Tension
}

Nilesh Badwe ${ }^{\mathrm{a}}$, Xiying Chen ${ }^{\mathrm{a}}$, Karl Sieradzki ${ }^{\mathrm{a}}$

a. Ira A. Fulton School of Engineering, Arizona State University, Tempe, Arizona 85287, USA

Corresponding Author:

Karl Sieradzki, PO Box 876106, Tempe, AZ 85287-6106, USA

Email: Karl.Sieradzki@asu.edu

Phone: 480-965-8990 Fax: 480-727-9321

\begin{abstract}
We report results of the tensile properties of nanoporous gold (NPG) as a function of the density and average ligament diameter. As-dealloyed tensile samples were thermally treated to coarsen the length scale of the NPG structure while increasing the sample density resulting from thickness reductions. The behaviors of samples with mean ligament diameters ranging from $30-$ $750 \mathrm{~nm}$ and corresponding densities ranging from $0.30-0.57$ that of bulk gold were examined. Digital image analysis was used to obtain ligament size histograms that were fit to the Weibull distribution. The Young's modulus was found to obey a power law, but with an exponent larger than that predicted by Gibson-Ashby scaling. The fracture behavior showed a brittle-ductile transition as a function of increasing ligament size. For samples characterized by a mean ligament diameter less than $\sim 220 \mathrm{~nm}$, the tensile behavior was linear elastic to sample fracture while samples with larger scale ligaments showed macroscopic yielding prior to fracture. These results are interpreted within the framework of extreme value statistics.
\end{abstract}


Keywords: Nanoporous gold; Mechanical properties; Digital image analysis; Brittle-to-ductile transition; Extreme value statistics.

\section{Introduction}

Nanoporous gold (NPG) is typically made from a binary alloy such as Ag-Au within a compositional range of $60-80 \mathrm{at} \% \mathrm{Ag}$. The lower bound corresponds to the so-called parting limit for this binary alloy system below which it is not possible to electrochemically selectively remove Ag from the bulk of the alloy at ambient temperature[1,2]. Below this parting limit only surface dealloying occurs during which the surface enriches in $\mathrm{Au}$ and eventually passivates. The upper bound is connected to the site percolation threshold for face-centered cubic systems[3, 4]. Fully dealloying a silver-gold alloy with less than 20 at $\%$ Au results in mechanically disconnected regions yielding significant volume changes. The morphology of NPG is that of an isotropic interpenetrating solid/void composite where both the solid and void phase are continuous throughout the bulk of the solid. This structure is often characterized by a mean ligament and/or pore diameter. Nevertheless the structure is random in the sense that there is a distribution in ligament and pore size that can be expected to control many aspects of the mechanical behavior of NPG that depend on extreme values within this distribution.

High-rate dealloying often results in damaged NPG structures. This damage takes the form of cracks that form in order to accommodate the volume changes which can occur during dealloying. In parent-phase polycrystalline alloys, the cracks are predominantly intergranular. This damage, if severe enough, can certainly affect measurements of the elastic, plastic and fracture properties of NPG samples. In the past 10 years various experimental protocols have been developed in order to obtain so-called "crack-free" NPG samples and all of these involve 
low-rate dealloying[5-7]. Presumably, dealloying at low rates allows for surface diffusional accommodation of volume changes that would otherwise occur at higher dealloying rates.

Various aspects of the mechanical properties of NPG have been experimentally studied in bending $[8,9]$, compression[10, 11], tension[12, 13], indentation[9, 14] and non-contact ultrasonics[15]. Computational studies have used mechanics of materials approaches based on a "unit-cell" of the NPG structure[16-18], finite element methods (FEM) [16, 19] and molecular dynamics (MD) [20-22]. The mechanics of materials models correspond to more detailed analyses but similar to the well-known Gibson-Ashby analysis which considers that a porous cellular solid can be represented by a unit cell of simply supported beams that respond to mechanical loading. In principle, such mechanics of materials based models can make accurate predictions for the elastic as well as the plastic behavior of porous solids so long as the degree of cell-to-cell randomness within the solid is not too large; in other words, so long as a useful representative volume element (RVE) can be identified. If on the other hand, there are significant levels of randomness (e.g., cell dimensions, beam dimensions comprising the cells, node points characterizing joints among beams, density fluctuations, etc.) within the porous solid then a suitable RVE may become difficult or impossible to identify. NPG solids likely correspond to this latter case. Both FEM and MD studies of NPG may suffer from this same limitation since in effect a RVE is always chosen for analysis. Nevertheless certain mechanical properties such as the elastic behavior of NPG may be accurately modeled using a suitable mean-field parameter such as the density. However, properties such as plastic yielding and fracture that depend on high order moments within the statistical distribution of ligament/pore sizes may not be accurately assessed using such approaches. These properties depend on the extreme values (e.g., the 
smallest diameter/length ligaments) within the tail of the distribution. In principle if one could experimentally characterize this region of the distribution, yielding and fracture behavior in these structures could be modeled. Ligament and pore size distributions of NPG can be determined with digital image analysis techniques, but even in this case one or many images of the structure that have been characterized in this way are likely not to yield sufficient information regarding the tail of the distribution. NPG morphologies have been produced with average ligament sizes ranging from about $5-1000 \mathrm{~nm}$. If we consider a cube of NPG of dimensions $1 \mathrm{~mm} \times 1 \mathrm{~mm}$ x 1 $\mathrm{mm}$, such a cube will contain about $10^{15}$ and $10^{8}$ ligaments respectively. We know of no experimental approaches for characterizing the extreme values within a solid containing such large populations of ligaments. However, toy models can be constructed by assuming certain forms for the distribution and many realizations of populations within the distribution can be used to at least qualitatively elucidate the physics controlling the break down behavior of such solids[23, 24].

One of our main motivations for this work was to characterize the elastic properties of NPG as a function of the density and the ligament size. In previous work Li and Sieradzki [8] were not able to get reliable results for the elastic properties because digital image correlation techniques were not as well developed and readily available as they are today. Additionally owing to the great improvements in the resolution of scanning electron microscopes in the past 25 years we were motivated to closely examine ligament size distributions in NPG structures and connect these to fracture behavior.

\section{Brief Review of Research aimed at Understanding the Mechanical Properties of NPG.}


Here we describe a set of experiments aimed at measuring the mechanical properties of NPG in tension as a function of the density and the average ligament/pore size. In order to place this work into context, we present a brief and by no means comprehensive review of the different experimental and computational approaches that have been employed to elucidate the mechanical properties of NPG. We focus on work that intersects with the experiment and analysis presented in sections 3 and 4 .

\subsection{Experiment.}

One of the most reliable methods for the determination of the elastic properties of a solid is through the measurement of the longitudinal and shear-wave velocities and using well-known relationships to calculate the elastic constants. Ahn and Balogun made such measurements using a laser-based ultrasonic technique[15]. For NPG samples with an average ligament size, $L$, in the range of $30-50 \mathrm{~nm}$, they obtained values of Young's modulus, $E^{N P G}$, in the range of $2.6-2.9$ GPa and Poisson's ratio in the range of $0.21-0.24$. They were also able to fit their results for $E^{N P G}$ to the Ashby-Gibson scaling form, $\left(E^{N P G} / E^{o}\right) \sim\left({ }^{N P G} /{ }^{o}\right)^{n}$ and obtained values of $n$ in the range of $2.5-3.0$. Here, $E^{o}$ and $\rho^{o}$ correspond respectively to the Young's modulus and density of bulk gold. Briot et al. reported on tensile test results for single crystal NPG with mean ligament size in the range of $32-60 \mathrm{~nm}[12]$. Within this range in ligament size, they found an average value of $E^{N P G}$ equal to $4.5 \mathrm{GPa}$ and Poisson's ratio of $0.22 \pm 0.06$. They reported that there was no obvious correlation between the ligament size and the elastic properties. In these same tests they measured the coincident yield and fracture stress and reported and an average 
value of 25.2 MPa. Mather and Erlebacher used a thin film technique involving the compressive deformation of $100 \mathrm{~nm}$ thick NPG leaf adhered to a thicker more compliant substrate[25]. An applied in-plane compression results in a buckling instability of fixed wavelength from which $E^{N P G}$ was determined. In this manner, they measured $E^{N P G}$ as a function of mean ligament size. As the ligament size increased from $3-40 \mathrm{~nm}, E^{N P G}$ decreased from about 40 to $6 \mathrm{GPa}$. One parameter that enters into the calculation is the thickness of the NPG leaf and they attributed potential errors in their measurements to errors in the thickness measurements. Li and Sieradzki examined the fracture behavior of $2 \mathrm{~mm} \times 2 \mathrm{~mm} \times 30 \mathrm{~mm}$ NPG beams in three-point bending as a function of mean ligament/pore size[8]. The NPG length scale was varied by thermally coarsening the as-dealloyed morphology. The main result of this investigation was the observation of a brittle to ductile transition with increasing mean ligament size. This transition occurred for a mean ligament size of $\sim 250 \mathrm{~nm}$. Below this size the samples were macroscopically brittle and above this size the samples were ductile.

\subsection{Computational Modeling}

Farkas et al. used a phase-field model to generate a bi-continuous NPG structure characterized by an average ligament size, $L$, of $1.8 \mathrm{~nm}$ and $\left({ }^{N P G} /{ }^{o}\right)=0.25[21]$. They modeled atomic scale interactions with a Johnson Embedded Atom Method (EAM) potential. Simulations were performed using periodic boundary conditions at a temperature of $300 \mathrm{~K}$. They obtained a value of $E^{N P G}$ of $3.70 \mathrm{GPa}$, a yield stress of $175 \mathrm{MPa}$ in tension and $25 \mathrm{MPa}$ in compression. This tension-compression asymmetry in yielding was attributed to surface stress effects. Ngo et al., used MD to study Young's modulus and yielding of NPG in compression and compared these results to their own experiments[22]. They used a Monte Carlo scheme simulating spinodal 
decomposition to evolve a NPG structure, with $L=3.15 \mathrm{~nm}$, and initial value of $\left({ }^{N P G} /{ }^{\circ}\right)=0.30$. A standard EAM potential was used to model the atomic scale interactions at $300 \mathrm{~K}$. In analogy with experiments, they performed a series of load/unload sequences and determined $E^{N P G}$ and yield behavior as a function of the degree of densification. Prior to densification they found a value of Young's modulus in compression of $280 \mathrm{MPa}$ that increased significantly with densification. The MD simulations mirrored their experimental results, however, it is important to note that while the relative density of the NPG in the experiments was similar to that in their simulation, the mean ligament size in experiment was $\sim 40 \mathrm{~nm}$. After ruling out a number of explanations for the anomalously low initial compliance of NPG in compression, the authors focus on surface stress induced bulk shear stress in the smallest ligaments within the distribution which they argued might result in a vanishing low shear modulus. In a latter paper from this same group they speculate that the anomalous compliance in compression may result from unconnected or dangling ligaments within the porous structure effectively reducing the load bearing components in the structure[26]. They acknowledge that a high population of dangling ligaments would be required in order to explain the low compliance. We note that to our knowledge no such anomaly has been reported in tension. Sun et al. used a phase field model that mimics spinodal decomposition to develop the atomic structure of NPG and performed MD simulations that incorporated a standard EAM potential[20]. They investigated the behaviors at fixed $L=3.26 \mathrm{~nm}$ while varying $\left({ }^{N P G} /{ }^{o}\right)$ between $0.24-0.36$ and at fixed $\left({ }^{N P G} /{ }^{\circ}\right)=0.30$ while varying $L$ between $2.45-4.08 \mathrm{~nm}$. For all these variations $E^{N P G}$ varied between $2.83-4.88 \mathrm{GPa}$. At fixed $L=3.26 \mathrm{~nm}$, they found that a modified Gibson 
Ashby scaling law of the form $E^{N P G} / E^{o}=C_{1}\left({ }^{N P G} /{ }^{o}\right)^{2}+C_{2}\left({ }^{N P G} /{ }^{o}\right)$ fit their results where the linear term accounted for the extensional deformation of the ligaments. At fixed density there appeared to be a weak dependence of the modulus on the average ligament size but this was difficult to confirm given the narrow range in $L$ examined and the standard deviations connected to the modulus measurements.

\section{Experimental Methods}

Silver-gold alloy sheets, $125 \mu \mathrm{m}$ in thickness of composition $\mathrm{Ag}_{0.70} \mathrm{Au}_{0.30}$ were cut using electric discharge machining to obtain $3 \mathrm{~mm}$ wide $\times 15 \mathrm{~mm}$ long samples. These samples were polished using 800 and $1200 \mathrm{SiC}$ paper and then annealed at $900 \mathrm{C}$ for 3 hours to relieve residual stresses. Samples were then placed on a thin gold film deposited on a mica substrate that served as a current collector during electrochemical dealloying. In order to fix the sample to the film, a thin gold wire was lightly wrapped around a sample and the film, to ensure electrical contact. The samples were immersed in $1 \mathrm{M} \mathrm{HNO}_{3}$ and potentiostatically dealloyed at $1.175 \mathrm{~V}$ vs. a normal hydrogen electrode. Dealloying was carried out until the current dropped below $3 \mu \mathrm{A}$ to ensure complete dealloying. The samples were dried and ligament-coarsening heat treatments were performed according to a previously published protocol [8]. Briefly, NPG samples were sandwiched between two thin quartz plates to ensure uniform heating in a furnace. Coarsening was carried under ambient atmospheric conditions at 400, 500, 600, and 700 ${ }^{\circ} \mathrm{C}$ for 15 minutes to obtain approximate ligament sizes of $110,225,400$ and $600 \mathrm{~nm}$. A 
ligament size larger than $700 \mathrm{~nm}$ was achieved through 20 minutes of heat treatment at $700{ }^{\circ} \mathrm{C}$.

For digital image correlation (DIC), the samples were decorated with a random speckle pattern using an airbrush (Iwata-Medea Eclipse HP CS Dual Action Airbrush Gun / Gravity Feed). A homemade horizontal micromechanical system was used for the mechanical testing. The samples were glued onto the aluminum loading platens. Tensile tests were then carried out by moving the stage in $3-5 \mu \mathrm{m}$ increments and capturing images using a Nikon D3200 (24 MP) camera fitted with a $49 \mathrm{~mm}$ extension tube-set and $18-55 \mathrm{~mm}$ f/3.5-5.6 AF-S DX VR NIKKOR Zoom Lens. DIC was used to determine particle displacements and corresponding strains. For each sample investigated, two load - unload curves were performed within the linear elastic region prior to loading the sample to fracture.

Ligament size distributions were determined using Image $\mathbf{J}$ software[27]. As illustrated in Figure 1, SEM images displaying a significant number of ligaments with high resolution were captured. An image was initially converted into 8-bit image. A binary image (image B) was then created using a threshold technique and a copy of this binary image (image C) was created. A distance map transformation was applied to one of the two binary images. This generates a Euclidian distance map (image D) where each foreground pixel in the binary image is replaced with a gray value equal to that pixel's distance from the nearest background pixel. The other binary image was skeletonized (image $S$ ) where center pixels of the ligaments were replaced with 1s (ones) and the rest of the pixels were made 0s (zeros). An image calculator was used to multiply image $\mathrm{D}$ and image $\mathrm{S}$ to yield an image $\mathrm{E}$. This image is similar to image $\mathrm{S}$, however 
now all the $1 \mathrm{~s}$ in the image $\mathrm{S}$ are replaced by the corresponding values from image $\mathrm{D}$ which corresponds to the distance of those pixels from the nearest black region. This represents half diameter of the ligament. Hence, image $\mathrm{E}$ is multiplied by 2 to obtain a final image, F. All the non-zero values in the image $F$ represent the diameter of the ligament at that particular location.

\section{Results and Analysis}

\subsection{Characterization of NPG samples}

Figure 2 shows SEM images of the as-dealloyed NPG sample surfaces and representative images of sample surfaces that were heat treated for the indicated temperature and times. The coarsening treatments we employed resulted in some degree of densification appearing as a change in thickness of the tensile samples and this allowed us to access the effect of density on the elastic properties. We noticed that some grain boundary cracking occurred on the surfaces of samples treated at temperatures of $500{ }^{\circ} \mathrm{C}$ and higher. It is difficult to assess how these defects affected the yield and fracture behavior of the tests described below, but their density was likely too low to have significantly affected the Young's modulus behavior.

Figure 3 and Supplementary Figure S1 show histogram results obtained from the DIA. These histograms are based on SEM micrographs of the outer sample surfaces since an analysis based on fracture surfaces would in our view be less accurate owing to ligament deformation and overlap. Supplemental Figure S2 compares SEM micrographs at the same magnification of the outer sample surfaces and the fracture surfaces, and shows no significant difference in ligament 
size. The red curves of Figures 3 and S1 correspond to a fit of the histogram data to the Weibull probability density function (PDF),

$$
\left.f(L,, k)=\frac{k}{(L}\right)^{k} \exp (\underline{L})^{k}
$$

where, $L$ is the ligament diameter, $k$ is called the shape parameter and $\lambda$ the scale parameter. In the Weibull distribution, the mean value, $L$, and standard deviation, $S D$, are given by,

$$
L=\left(1+\frac{1}{k}\right)
$$

and

$$
S D=\left\{\left(1+\frac{2}{k}\right)\left[\left(1+\frac{1}{k}\right)\right]^{2}\right\}^{1 / 2}
$$

where $\Gamma(x)$ is the gamma function. A statistical analysis of all the values of the shape parameter yields a mean value of 2.87 and a median of 2.86. The PDFs are symmetrical and as shown in Figure 3d nearly self-similar. Supplementary Figure S3 shows results for standard deviations versus mean values and this further confirms the self-similarity of the PDFs.

\subsection{Elastic properties of NPG as a function of density and ligament size}

Figure 4a shows representative stress-strain curves for the NPG samples. Typically samples with a mean ligament diameter less than $\sim 220 \mathrm{~nm}$ showed linear elastic behavior to fracture with 
no detectable macro-scale plastic yield behavior. The Young's modulus, $E^{N P G}$, and Poisson's ratio, $v$, for each sample was determined using DIC from a series of load-unload curves.

We examined how these elastic constants varied with sample density, $\rho$. The sample density was evaluated using two measures. One was a global measure based on the gold composition of the alloy and the overall sample dimension. For the as-dealloyed NPG, the tensile samples contained 1 at $\% \mathrm{Ag}$ or less so that the density of this was taken to be 0.30 that of bulk gold. As described earlier, coarsening treatments resulted in thickness reductions of the tensile samples that allowed us to determine the density. Another measure that we used to evaluate an effective density was the square of the ratio of mean ligament diameter, $L$, to the mean pore size, $L_{\text {pore }}$. The mean pore size is a measure of the "cell size" in the structure so that the quantity $\left(L / L_{\text {pore }}\right)^{2}$ corresponds to a scaled microscopic measure of the density. We note that this measure can be subject to considerable error if there are large density gradients within the coarsened NPG structures. Our data revealed no simple correlation for Poisson's ratio with either $\rho$ or $L$ that varied between $0.18-0.28$. We note that for open cell structures Poisson's ratio should not be a function of density and only be a function of cell geometry[28]. Figure 4b shows the Young's modulus behavior as a function of the density and Supplementary Figure S4 shows the same data on a log-log plot. The error bars correspond to variations in the thickness of a particular sample causing uncertainties in both the density and the stress. Unfortunately, there is a density gap in the data in the vicinity of $\left({ }^{N P G} /{ }^{o}\right) \sim 0.4$. This occurred because our coarsening treatments were aimed at obtaining a target ligament diameter rather than a particular density. Nevertheless, 
we fit this data to variants of the general form $E^{N P G} / E^{o}=C_{1}\left({ }^{N P G} /{ }^{o}\right)^{n}+C_{2}\left({ }^{N P G} /{ }^{o}\right)+C_{3}$. The best fit that we found was for a pure power law, with $n=2.8$ and $C_{1}=0.86, C_{2}=C_{3}=0$. A power-law fit with $n=2.0, C_{2}=C_{3}=0$ yielded a significantly poorer quality of fit. We also tried a more general form, expected from Gibson-Ashby scaling, for systems displaying compliance contributions from both ligament bending and stretching, and obtained a good fit with a value of $n=2.0, C_{1}=0.66$ and $C_{2}=-0.10, C_{3}=0$, however, the negative value of $C_{2}$ makes this result difficult to interpret. There is a good linear fit to the data, with $C_{1}=0, C_{2}=0.42$ and $C_{3}=-0.10$. Extrapolation of these fits to full density gold indicates that the simple $n=2.8$ power law is the best fit to our data. Supplementary Figure S5 shows the behavior of Young's modulus as a function of the effective density parameter, $\left(L / L_{\text {pore }}\right)^{2}$, for which we observe power-law scaling with an exponent of 3.1 .

\subsection{Fracture of $N P G$}

In computer simulations of fracture of disordered or so-called network solids the terms ductile and brittle refer to the nature of the damage introduced in the system during loading. If many distributed cracks form within the sample prior to fracture, the load-displacement curve is nonlinear and the system behavior is termed ductile. On the other hand, if only a "few" distributed cracks form prior to the formation of a dominant crack then the load displacement curve will be linear to fracture and the system behavior is termed brittle. Once a dominant crack forms, ligament fracture is correlated[13]. The term dominant crack refers to a crack of sufficient size so that its stress/strain concentration factor results in crack tip stresses/strains larger than that required to break the strongest ligament within the distribution[23]. This means that the 
ligaments in front of a dominant crack essentially fracture one after another resulting in a relatively straight crack path on the scale of the ligament size. In a computer simulation these behaviors are often quantified by an order parameter, $n_{b}=N_{b} /(X / L)^{d}$, where $N_{b}$ is the number of broken ligaments that form just prior to fracture, $d$ refers to the dimension of the sample, and $X$ is the actual sample-size perpendicular to the loading direction[23,29]. For a two-dimensional sample, completely brittle behavior is characterized by $n_{b} \rightarrow 0$, i.e., fracture of a single ligament corresponds to the formation of a dominant crack. This can only occur for a very narrow distribution in ligament strength or diameter. For ductile behavior, $n_{b} \sim(X / L)^{y}$, where, $y$ is between 0 and 1 . In experiments on NPG, $N_{\mathrm{b}}$ cannot be measured, but we make the ansatz that the fracture strain should be a quantity connected to this order parameter. Also, what distinguishes the behavior of NPG from the model systems examined in computer simulation is that in NPG all ligaments fail in a ductile manner by dislocation-mediated shear. Given this, it is somewhat remarkable that these structures can show linear load-displacement behavior in tension to sample fracture.

The tensile samples were pulled to fracture and we examined the behavior of the yield strength, $\sigma_{y}$, fracture strength $\sigma_{f}$, and fracture strain, $\varepsilon_{f}$. As described in an earlier publication, we use the reciprocal of the natural length scale in these nanostructures, $1 / L$, as a parameter characterizing the effective or scaled sample size[8]. Assuming that the yield and fracture behavior is controlled by extreme value statistics of the Weibull type, one expects to observe power-law behavior in these parameters as a function of sample size. We consider the cumulative Weibull distribution in the form, 


$$
F\left[x,(1 / L)^{d}\right]=1 \quad \exp \left((1 / L)^{d}\left(\frac{x}{-k}\right)^{k}\right)
$$

where $x$ pertains to $\sigma_{y}, \sigma_{f}$, or $\varepsilon_{f}$. When comparing two samples of different size with the same probability of failure, one can show that $x \sim(1 / L)^{d / k}[30]$. The average of the shape parameter, $<k>$, of samples displaying linear stress-strain behavior to fracture is 2.91 so that for $d=3$, $-d /<k>\cong-1.03$ and for $d=2,-d /<k>\cong-0.69$. We have tested this prediction by plotting the fracture strain as a function of $(1 / L)$. Figures $3 \mathrm{c}$ and $3 \mathrm{~d}$ show this behavior for $\varepsilon_{f}, \sigma_{y}$ and $\sigma_{f}$. For samples that displayed no yielding in the stress-strain curve we assume that the macroscopic yield and fracture stress are concomitant. The fracture strain behavior shows two regimes of behavior as a function of $(1 / L)$. As the scaled sample size increases the fracture strain first increases and then decreases. We term the behavior where $\varepsilon_{f}$, increases as ductile and that where $\varepsilon_{f}$ decreases with $(1 / L)$ as brittle. The fracture behavior of the NPG within the brittle regime is typical of sample size effects controlled by extreme value statistics. In this brittle regime we observe power-law behavior with an exponent of -0.61 , close to the expected value for an effective 2D sample. This indicates that the dominant crack forming in the brittle regime is likely a through-thickness planar defect. In the ductile regime of behavior, the sample does not contain enough ligaments (i.e., the scaled sample size is not large enough) to develop a dominant crack and sample fracture is determined by uncorrelated ligament fracture. Thus in this regime the fracture strain should increase with the effective sample size as there is a greater percentage of ligaments that have to fail prior to sample fracture. As shown in Figure 5, this general scenario is 
reflected in the appearance of the fracture surfaces, which are relatively flat for samples displaying brittle behavior and rough for samples displaying ductile behavior.

\section{Discussion}

Owing to the nanoscale size of ligaments in NPG structures, one may be tempted to analyze the macro-scale yield behavior we observed in terms of size effects in plasticity or the "smaller is stronger" concept[31-33]. Certainly the yield behavior of individual ligaments within the structure depends on size [34]. However, if one accepts the premise that the network structure behaves as a quasi-brittle material (for small enough L), then the fracture behavior is controlled by the weakest-link hypothesis. Since ligament yielding precedes fracture, the weakest link scenario must also control yield behavior. Figure $4 \mathrm{~d}$ shows the macro-scale yield stress as a function of $1 / \mathrm{L}$ and does not show the behavior that one would expect based on the smaller is stronger concept.

The scaling results for the fracture stress obtained in this investigation closely mirrored that obtained previously in bending. Li and Sieradzki found a scaling exponent of -0.2 in that work[8] and herein we report an exponent of -0.23 . The scaling exponent on the fracture strain $(-0.69)$ indicates that within the brittle regime of behavior, the Weibull shape parameter should be 2.89 , which is close to the average value of the shape parameter (2.87) for the brittle NPG samples. 
Our biggest surprise in this investigation was the magnitude of the shape parameter obtained by fitting the ligament size histograms to the Weibull distribution. The relatively small value of the shape parameter is indicative of a wide distribution in ligament size and this is reflected by the large value of the standard deviation obtained for all the NPG structures examined. Our surprise is that such a wide distribution in ligament size can result in macroscopically brittle behavior. Apparently the physical size of the tensile samples we examined is large enough to result in brittle behavior of NPG characterized by a mean ligament diameter less than $\sim 220 \mathrm{~nm}$. Our results imply that if the overall sample dimensions were increased by about a factor of 10 , NPG with a mean ligament diameter of $\sim 600 \mathrm{~nm}$ would show macroscopically brittle behavior. Unfortunately this would seem to be a difficult prediction to test, as it is difficult to produce crack-free samples of NPG this size. However, it would seem possible to test this prediction by examining NPG samples of mean ligament diameter in the range of $150-200 \mathrm{~nm}$ using samples about one-tenth the size of the current samples. For samples such as this we would expect to see ductile behavior in the load displacement curve.

We believe that there are several ways for which the current investigation could be improved. First instead of following previous protocols for coarsening the as-dealloyed NPG structure, lower temperature treatments over considerably extended times should be employed. We have performed some ancillary work for coarsening NPG structures at temperatures below $500{ }^{\circ} \mathrm{C}$ and found that the surface cracking we observed in some of our coarsened structures could be avoided for lower temperature treatments and times extending as much as 10 days. Secondly, non-contact ultrasonic techniques could be used as an additional means of measuring the elastic constants within the isotropic NPG structures. Such work may lead to a clarification of the 
reported anomalous elastic behavior of as-dealloyed NPG structures in compression. Third, focused ion beam serial sectioning of the NPG morphologies could be used to provide 3D characterization of the nanostructures using DIA.

\section{Conclusions}

In this study we examined the tensile properties of NPG as a function of mean ligament diameter (ranging from $30-750 \mathrm{~nm}$ ) and corresponding density (ranging from $0.30-0.57$ that of bulk gold). These parameters were varied by thermal treatment of electrochemically as-dealloyed samples. The following major conclusions emerged from this work:

- Digital image analysis shows that for the range of mean ligament size examined the ligament distributions are nearly self-similar and show a standard deviation equal to about 0.35 .

- The Young's modulus behavior as a function of density displayed power-law behavior with a scaling exponent between $2.8-3.1$. We found no correlation between the NPG sample density and Poisson's ratio that varied between $0.18-0.28$.

- The fracture behavior of NPG showed a brittle to ductile transition as a function mean ligament size, $L$. For $L$ less than $\sim 220 \mathrm{~nm}$ the load-displacement behavior was linear elastic to sample fracture while for larger $L$ we observed macroscopic ductility in the tensile tests. Within the brittle regime of behavior we found that the fracture strain, ${ }_{f}$,

scales with $L$ as ${ }_{f} \sim(1 / L)^{0.61}$. This scaling is consistent with that expected for brittle 
solids based on the concept of the weakest link and the Weibull extreme value distribution.

\section{Acknowledgement}

The authors are grateful for the support of this work by the US DOE Basic Energy Sciences under award DE-SC0008677. NB thanks Dr. James Mertens for help with digital image analysis protocol.

\section{References}

[1] J. Erlebacher, M.J. Aziz, a Karma, N. Dimitrov, K. Sieradzki, Evolution of nanoporosity in dealloying., Nature 410 (2001) 450-453.

[2] H.W. Pickering, Characteristic features of alloy polarization curves, Corros. Sci. 23 (1983) 1107-1120.

[3] D. Stauffer, A. Aharony, Introduction to Percolation Theory, Second., Taylor \& Francis, London, Washington, DC, 1992.

[4] K. Sieradzki, Curvature effects in alloy dissolution, J. Electrochem. Soc. 140 (1993) $2868-2872$.

[5] N. a Senior, R.C. Newman, Synthesis of tough nanoporous metals by controlled electrolytic dealloying, Nanotechnology 17 (2006) 2311-2316.

[6] Y. Sun, T.J. Balk, A multi-step dealloying method to produce nanoporous gold with no volume change and minimal cracking, Scr. Mater. 58 (2008) 727-730.

[7] H.J. Jin, L. Kurmanaeva, J. Schmauch, H. Rösner, Y. Ivanisenko, J. Weissmüller, Deforming nanoporous metal: Role of lattice coherency, Acta Mater. 57 (2009) 26652672. 
[8] R. Li, K. Sieradzki, Ductile-brittle transition in random porous Au, Phys. Rev. Lett. 68 (1992) 1168-1171.

[9] E.-J. Gwak, J.-Y. Kim, Weakened flexural strength of nanocrystalline nanoporous gold by grain refinement, Nano Lett. 16 (2016) 2497-2502.

[10] H.-J. Jin, J. Weissmüller, A material with electrically tunable strength and flow stress., Science 332 (2011) 1179-1182.

[11] N. Mameka, J. Markmann, H.J. Jin, J. Weissmüller, Electrical stiffness modulation Confirming the impact of surface excess elasticity on the mechanics of nanomaterials, Acta Mater. 76 (2014) 272-280.

[12] N.J. Briot, T. Kennerknecht, C. Eberl, T.J. Balk, Mechanical properties of bulk single crystalline nanoporous gold investigated by millimetre-scale tension and compression testing, Philos. Mag. 94 (2014) 847-866.

[13] S. Sun, X. Chen, N. Badwe, K. Sieradzki, Potential-dependent dynamic fracture of nanoporous gold, Nat. Mater. 14 (2015) 894-898.

[14] J. Biener, A.M. Hodge, A. V. Hamza, L.M. Hsiung, J.H. Satcher, Nanoporous Au: A high yield strength material, J. Appl. Phys. 97 (2005) 2-5.

[15] P. Ahn, O. Balogun, Elastic characterization of nanoporous gold foams using laser based ultrasonics, Ultrasonics 54 (2014) 795-800.

[16] N. Huber, R.N. Viswanath, N. Mameka, J. Markmann, J. Weissmüller, Scaling laws of nanoporous metals under uniaxial compression, Acta Mater. 67 (2014) 252-265.

[17] R. Liu, A. Antoniou, A relationship between the geometrical structure of a nanoporous metal foam and its modulus, Acta Mater. 61 (2013).

[18] R. Xia, X.-Q. Feng, G.-F. Wang, Effective elastic properties of nanoporous materials with hierarchical structure, Acta Mater. 59 (2011) 6801-6808.

[19] H.-H. Cho, Y.K. Chen-Wiegart, D.C. Dunand, Finite element analysis of mechanical stability of coarsened nanoporous gold, Scr. Mater. 115 (2016) 96-99.

[20] X.-Y. Sun, G.-K. Xu, X. Li, X.-Q. Feng, H. Gao, Mechanical properties and scaling laws of nanoporous gold, J. Appl. Phys. 113 (2013) 23505.

[21] D. Farkas, A. Caro, E. Bringa, D. Crowson, Mechanical response of nanoporous gold, Acta Mater. 61 (2013) 3249-3256.

[22] B.N.D. Ngô, A. Stukowski, N. Mameka, J. Markmann, K. Albe, J. Weissmüller, Anomalous compliance and early yielding of nanoporous gold, Acta Mater. 93 (2015) 144-155.

[23] B. Kahng, G.G. Batrouni, S. Redner, L. de Arcangelis, J. Herrman, H, Electrical breakdown in a fuse network with random, continuously distributed breaking strengths, Phys. Rev. B 37 (1988) 7625-7637. 
[24] P.M. Duxbury, P.D. Beale, P.L. Leath, Size effects of electrical breakdown in quenched random media, Phys. Rev. Lett. 57 (1986) 1052-1055.

[25] A. Mathur, J. Erlebacher, Size dependence of effective Young's modulus of nanoporous gold, Appl. Phys. Lett. 90 (2007) 1-4.

[26] N. Mameka, K. Wang, J. Markmann, E.T. Lilleodden, J. Weissmüller, Nanoporous gold -testing macro-scale samples to probe small-scale mechanical behavior, Mater. Res. Lett. 4 (2016) 27-36.

[27] W.S. Rasband, ImageJ [Software], U. S. Natl. Institutes Heal. Bethesda, Maryland, USA (2015) //imagej.nih.gov/ij/.

[28] L.J. Gibson, M.F. Ashby, cellular solids: Structure and Properties, 1st ed., Perganon Press, Oxford, 1988.

[29] P.M. Duxbury, P.L. Leath, P.D. Beale, Breakdown properties of quenched random systems: The random-fuse network, Phys. Rev. B 36 (1987) 367-380.

[30] A. Rinaldi, P. Peralta, C. Friesen, K. Sieradzki, Sample-size effects in the yield behavior of nanocrystalline nickel, Acta Mater. 56 (2008) 511-517.

[31] M.D. Uchic, D.M. Dimiduk, J.N. Florando, W.D. Nix, Sample dimensions influence strength and crystal plasticity, Science 305 (2004) 986-989.

[32] J.R. Greer, W.C. Oliver, W.D. Nix, Size dependence of mechanical properties of gold at the micron scale in the absence of strain gradients, Acta Mater. 53 (2005) 1821-1830.

[33] C.A. Volkert, E.T. Lilleodden, Size effects in the deformation of sub-micron Au columns, Philos. Mag. 86 (2006) 5567-5579.

[34] K. Sieradzki, A. Rinaldi, C. Friesen, P. Peralta, Length scales in crystal plasticity, Acta Mater. 54 (2006) 4533-4538.

\section{Figure Captions}

Figure 1. Schematic of digital image analysis protocol to obtain ligament size distribution.

Figure 2. Scanning electron micrographs of the as-dealloyed and thermally coarsened NPG structures at different levels of magnification. Samples have a grain size ranging from about 10 $50 \mu \mathrm{m}$. (a) as-dealloyed; scale bar $300 \mu \mathrm{m}$, (b) as-dealloyed; scale bar $10 \mu \mathrm{m}$ (c) as-dealloyed; 
scale bar $2 \mu \mathrm{m}$. (d) $L=45 \mathrm{~nm}$, as dealloyed; scale bar $500 \mathrm{~nm}$ (e) $L=125 \mathrm{~nm}, 400{ }^{\circ} \mathrm{C}, 15$ minutes; scale bar $1 \mu \mathrm{m}$ and (f) $L=590 \mathrm{~nm}, 700{ }^{\circ} \mathrm{C}, 15$ minutes; scale bar $5 \mu \mathrm{m}$.

Figure 3. Results of digital image analysis showing histograms for samples with mean ligament diameters, of (a) 45, (b) 223 (c) $589 \mathrm{~nm}$. (d) Normalized histograms where within each distribution the ligament size was normalized by the mean ligament diameter and the number of ligaments was normalized by the maximum count. The widest distribution is for the distribution that has an average ligament diameter of $713 \mathrm{~nm}$. The Weibull shape parameter, $k$ and the mean shape parameter $<k>$ for all the histograms are indicated.

Figure 4. Tensile behavior of NPG. (a) Representative stress-strain curves of 6 samples with varying mean ligament size: curve 1, $742 \mathrm{~nm}$; curve 2, $706 \mathrm{~nm}$; curve 3, $589 \mathrm{~nm}$; curve 4, 399 $\mathrm{nm}$; curve 5, $45 \mathrm{~nm}$; curve 6; $223 \mathrm{~nm}$. (b) Normalized Young's modulus versus the normalized NPG density where $\mathrm{E}^{\mathrm{o}}$ is the bulk modulus of gold (79 GPa) and $\square^{\circ}$ is the density of bulk gold, $19.3 \mathrm{gmcm}^{-3}$. The dashed curve is a fit to $E^{N P G} / E^{o}=C_{1}\left({ }^{N P G} /{ }^{o}\right)^{n}$ with $n=2.8$ and $C_{1}=0.86$, (c) and (d) Scaling behavior of yield and fracture of NPG. Double logarithmic plots of (c) the fracture strain and (d) the yield and fracture stress as a function of the effective sample size. In (d) the black dots correspond to the fracture stress and the red X's correspond to the yield stress. The red lines are power law fits for the behavior in the brittle regime for which ${ }_{f} \sim(1 / L)^{0.61}$ and $f(1 / L)^{0.23}$. The blue line in is a power law fit (circled points not included) in the ductile regime where ${ }_{f} \sim(1 / L)^{0.77}$. Error bars on the strain behavior are smaller than the symbols. Error bars on the stress, not shown for clarity, are connected to uncertainties in the sample thickness and correspond to $\pm 5 \%$ of the indicated values. 
Figure 5. Fracture surfaces of NPG samples showing how the surface roughness qualitatively varies with the mean ligament size in the structure. (a) $45 \mathrm{~nm}$, (b) $125 \mathrm{~nm}$ and (c) $589 \mathrm{~nm}$. All scale bars are $50 \mu \mathrm{m}$. 


\section{Skeletonize}

Image S - All pixel values

where $\mathrm{Au}$ is present $=1$

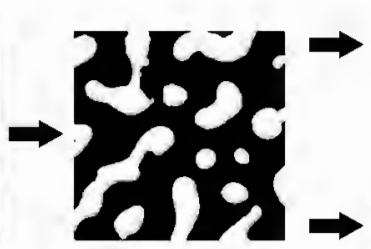

Binary image

Image B

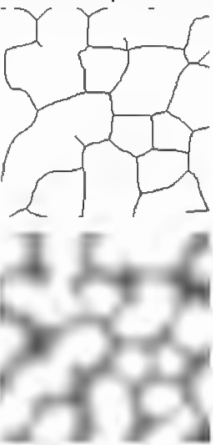

Distance map Image D

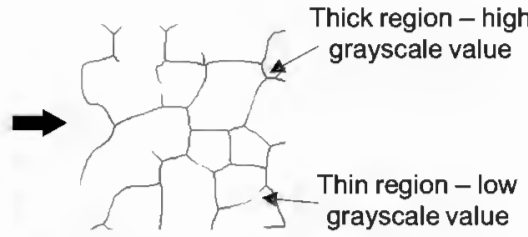

(Image D $\times$ Image $S=$ Image E) $\times 2$

Image $\mathrm{F}$ - All pixel values where $\mathrm{Au}$ is present = thickness of ligament 


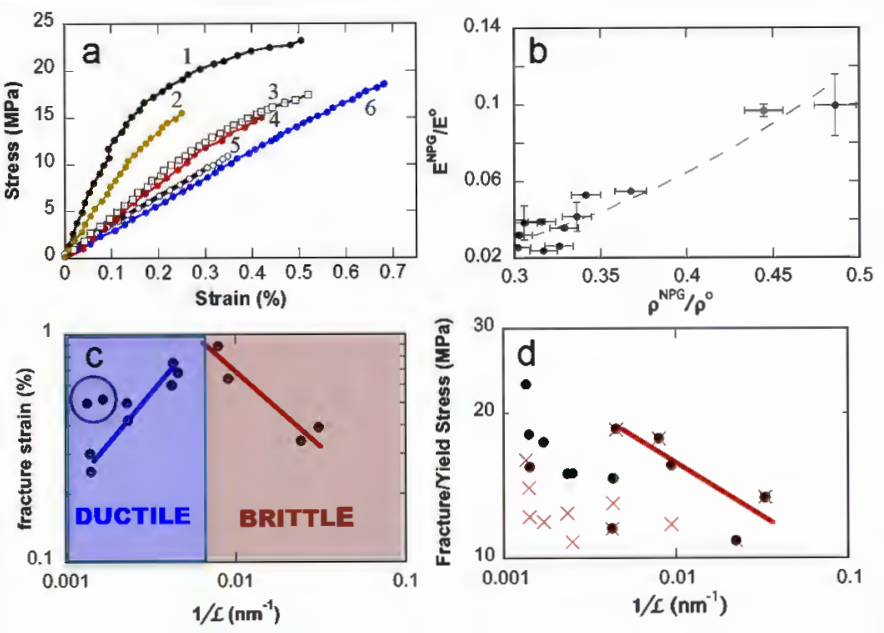




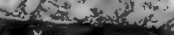

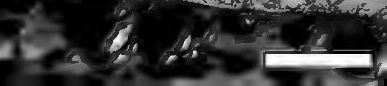



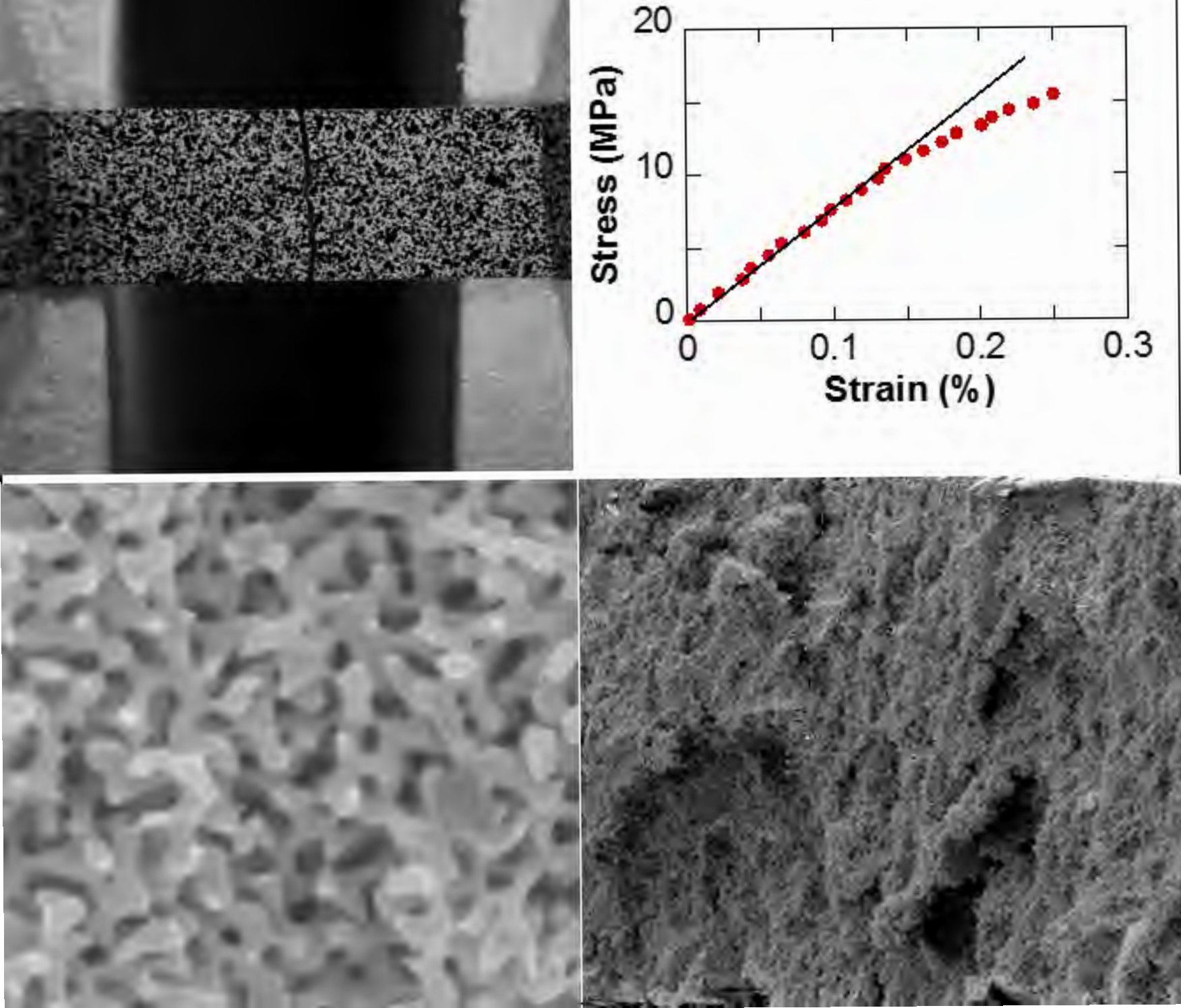